\title{
Sorafenib in metastatic MTC - a case report and minireview of the literature
}

This article was published in the following Dove Press journal:

International Medical Case Reports Journal

21 May 2010

Number of times this article has been viewed

\author{
Mila Petrova' \\ Zhasmina Mihaylova' \\ Albena Fakirova ${ }^{2}$ \\ 'Hematology and Oncology \\ Department, ${ }^{2}$ Pathology Department, \\ Medical Military Academy, \\ Sofia, Bulgaria
}

\begin{abstract}
Medullary thyroid cancer (MTC) is a rare and only surgically treatable disease with early development of metastases and bad prognosis. Due to the lack of efficient systemic treatment, new strategies and approaches are needed to better the patients' outcome. One of the most promising treatment options is the use of tyrosine multikinase inhibitors, which appear to have some effect on the disease progression with tolerable toxicity. Most of them affect many key pathways in the cancer genesis and have proven their effect in different malignancies. We report a case of a young patient with metastatic MTC treated successfully for two months with sorafenib.
\end{abstract}

Keywords: medullary thyroid cancer, tumor response, cardiotoxicity, TKIs

\section{Introduction}

Medullary thyroid cancer (MTC) remains a disease which can be treated only by surgery. Chemotherapy, radiation, and iodine captation have no place in the management of this rare disease. We report a case of a young woman with metastatic MTC who after only two months treatment with sorafenib experienced tumor response.

A 23-year-old female patient with no family history of multiple endocrine neoplasia (MEN) syndrome, familial MTC, or sporadic MTC was diagnosed with spontaneous metastatic MTC in March, 1993. The patient underwent total thyroidectomy and central neck lymph node dissection, revealing a mass of $3 / 2 \mathrm{~cm}$ in the right lobe and a similar $3 / 3 \mathrm{~cm}$ one in the left lobe without capsule involvement. One lymph node resected from the central neck prelaryngealis and two lymph nodes resected from the lateral neck trigonium (lateral from the $v$. jugularis interna dextra) revealed metastases with no extranodal extension. Chest or abdomen computed tomography (CT) were negative. The tumor was classified T3N1M0.

The patient remained disease-free until March, 2009 (16-year disease free period) when, because of a resistant inflammatory lung disease, a CT of the thorax and abdomen was presented. The imaging tests showed many small diffuse lesions in the lungs, adenopathy in the right lung hilus and in the mediastinum (at the bifurcation of the trachea mostly), small lesions in the liver, a lesion $48 / 59 \mathrm{~mm}$ in the left adrenal gland, as well as an osteolytic zone in the cortex of L4. Ultrasound-guided biopsy of the liver proved MTC (100\% expression of calcitonin and moderate expression of chromogranin A, sinaptophysin, and epithelial membrane antigen).

The patient was referred to our institution at the end of April, 2009. The initial laboratory results showed slightly elevated transaminases (ALT 46, AST 67), lactate
Correspondence: Mila Petrova

I Georgy Sofiisky Boulevard,

1606 Sofia, Bulgaria

Tel +359883 335955

Fax +359292 26072

Email milllapetrova@gmail.com 
dehydrogenase was 477, and the other routine blood parameters, including calcium level, were in reference. The calcitonin level was $5,503 \mathrm{pg} / \mathrm{mL}$ (normal up to $19 \mathrm{pg} / \mathrm{mL}$ ), thyreoglobulin level $0.01 \mathrm{ng} / \mathrm{mL}$ (normal 2-70 ng/mL), and the examined thyroid hormones revealed hypothyreoidism (Free F3: 1.00 and thyroid-stimulating hormone [TSH]: 21.4) while taking levothyroxine $1.75 \mu \mathrm{g}$ daily. From May 1st, 2009 the patient started therapy with sorafenib $2 \times 400 \mathrm{mg}$ daily and Zometa ${ }^{\circledR}$ (Novartis, Basel, Switzerland) intravenous every 28 days after a written consent from the patient was established. The dosage of levothyroxine was increased. No rearranged during transfection (RET) mutation examinations were done.

On May 25th, 2009 the patient appeared for a follow-up visit and an estimation of the adverse reactions. The main complaints were fatigue, anorexia, athralgia, and mialgia. Two days after the admission of sorafenib dry, exfoliative macular-like not-itching rash on the abdomen, palms, and feet appeared, and disappeared without any treatment. On both the elbows and knees, two exfoliations were present. The patient revealed that they experienced hand-foot syndrome grade 1 for only two days. Since the examination and the lab results (excluding the TSH level, which had fallen to 12.5 ) did not differ from the initial ones, the treatment continued.

On June 24th, 2009 (almost two months after the start of the treatment) the control $\mathrm{CT}$ revealed decrease in size and count of the lesions (liver, lung, and adrenal gland) and also reduction of the mediastinal lymph node metastases. The patient still experienced fatigue and persistent mialgia as well as intermediate moderate pains in the lumbar region, not connected with the position or the movement of the body. The lab results showed normal complete blood count, slightly elevated transaminases and cholestatic enzymes, and TSH level 10.2. The calcitonin level was 1,778 (almost three-fold decrease since the start of the treatment). The sorafenib treatment went on with the same dose as at the beginning.

Two weeks later we were informed about the sudden death of the patient at home. She had experienced strong fatigue and myalgia to a degree she could not move at all, strong anorexia and pains all over her body, and difficulty with breathing and chest pain. No tests were performed and the cause of the death remained unclear as no autopsy was established.

\section{Discussion}

Thyroid cancer is an uncommon disease, but it is the most common malignancy of the endocrine system. ${ }^{1}$ Differentiated carcinoma (ie, papillary, follicular, and their variants) and MTC represent the two most common subtypes, with differing etiologies, prognoses, and management strategies. ${ }^{2}$ While the first subtype is highly treatable and usually curable, the second one is more aggressive, metastasize early, and has a much poorer prognosis.

MTC is a neuroendocrine tumor arising from neuralcrest-derived, calcitonin-secreting parafollicular $\mathrm{C}$ cells within the thyroid. The disease accounts for $5 \%-10 \%$ of all thyroid cancers. ${ }^{3}$ MTC may occur either sporadically or as a part of a hereditary disease $(25 \%-30 \%)$ such as MEN Type 2A, MEN Type 2B, or familial MTC. ${ }^{4,5}$ Serum calcitonin is still the most specific and sensitive marker for MTC for both the primary diagnosis and postsurgical follow-up.

The primary treatment of MTC is surgical removal of all neoplastic tissue present in the neck and lymph nodes. ${ }^{6}$ Surgery is still the standard treatment since there is no known curative systemic treatment for this neoplastic disease. Unfortunately, at the time surgery is established many of the patients (around 50\%) develop metastases. For nonmetastatic patients, the 5- and 10-year results after surgery strongly depends on gender, tumor-affected organ, and surgeons' professional experience.

In regards to the other curative options it should be emphasized that MTC cells do not concentrate radioactive iodine and MTC does not respond well to the conventional cytostatic treatment or to the TSH suppression. MTC lacks effective systemic treatments. ${ }^{9-11}$

In recent years, however, huge advances have been made in understanding the molecular pathways and cellular pathogenesis of the disease. This knowledge has in turn led to the development of different targeted therapies - some of them specific to thyroid cancer genetic alterations such as the RET/papillary thyroid carcinoma translocation, while others focuses on general malignant properties such as angiogenesis, ${ }^{12}$ mainly the vascular endothelial growth factor (VEGF) and its receptor. For most of the authors the RET proto-oncogene plays a key role in the development of MTC. ${ }^{13}$ Initially, RET was thought to be found only in MTC patients with hereditary disease. After a while it was revealed that in nearly one half of the sporadic MTC cases RET mutation was present. ${ }^{14}$ The role of RET oncogenes and the encoded active oncoproteins as potential targets has been investigated by different strategies, but targeted treatment specifically for RET-driven cancers is not clinically available in current therapy. Small molecule tyrosine kinase inhibitors (TKIs), including sorafenib, sunitinib, motesanib and 
vandetanib, which have already shown efficacy against other neoplastic diseases, are being evaluated in clinical trials for treatment of MTC. Most of them, also described as RET inhibitors, are multikinase inhibitors with antiangiogenic activity related to inhibition of receptor tyrosine kinases, such as the VEGF receptors. ${ }^{15,16}$ These small molecules also inhibit many multiple intracellular and cell surface kinases (eg, c-Kit, Flt-3, platelef-derived growth faeta receptors [PDGFR]).

First results of "targeted therapies" in medullary and differentiated thyroid carcinoma are impressive: phase II trials targeting RET or VEGF receptor kinases show objective tumor response in up to $50 \%$ of the patients. ${ }^{14,17}$ The promising results also reveal generally tolerable toxicity. The most common treatment-related toxicities are rash, nausea, and diarrhea. ${ }^{17}$ The cardiotoxicity is uncommon, but when expressed may have a life-threatening consequences. It may range from asymptomatic subclinical abnormalities such as electrocandiogram (ECG) changes and left ventricular ejection fraction decline to life threatening events like congestive heart failure and acute coronary syndromes. ${ }^{18}$

The management of MTC with TKIs, particularly those affecting RET activity is promising and the initial trials show a certain benefit on their behalf. This shows a significant hope for patients with metastatic MTC. However, more randomized prospective clinical trials are needed to confirm the efficacy of the TKIs and they have to pay greater attention to the side effects, namely cardiotoxicity. As a result of this, in a few years TKIs eventually may prove worthy of regulatory approval for MTC.

In our case we presented a woman with metastatic MTC with good Eastern Cooperative Oncology Group (ECOG) performance status and relatively normal laboratory results, with the exception of the calcitonin level (the 16-year disease free period after the operation was remarkable and rather unexplainable for us). Due to the lack of a standard for treating these patients we started a therapy with sorafenib, a multityrosinekinase inhibitor which not only blocks the VEGF receptors, the PDGFR receptors, and c-Kit, but also has a role in inhibiting RET oncogens as well. Despite the short interval that we were able to follow her treatment, the CT results (based on the Response Evaluation Criteria in Solid Tumors (RECIST), the tumor rate diminished by almost $15 \%$ ) and the calcitonin level two months after the start of the treatment showed tumor response. Unfortunately, at that time the patient died suddenly. This may have been due to the rare, but significant, cardiotoxicity of the drug and the patient may have experienced sudden cardiac dysfunction (acute coronary syndrome/cardiac arrest). Or the sudden death may be due to the development of thrombosis and establishment of pulmonary embolism. Since the patient did not die in our institution and since the lack of any clinical, laboratory or imaging data, the cause of the death remains unclear and only a hypothesis could be developed.

Nevertheless we are convinced that our case supports the early trials' results that the use of small molecules TKIs may have an effect in this rare localization and it is worthy performing prospective randomized clinical trials. Unfortunately, we were unable to proceed with the treatment and make more detailed and firmer conclusions.

\section{Disclosures}

The authors report no conflicts of interest in this work.

\section{References}

1. Hundahl SA, Fleming ID, Fremgen AM, et al. A National Cancer Data Base report on 53,856 cases of thyroid carcinoma treated in the US 1985-1995. Cancer. 1998;83(12):2638-2648.

2. Ying AK, Huh W, Bottomley S, et al. Thyroid cancer in young adults. Semin Oncol. 2009;36(3):258-274.

3. Moo-Young TA, Traugott AL, Moley JF. Sporadic and familial medullary thyroid carcinoma: state of the art. Surg Clin North Am. 2009;89(5):1193-1204.

4. Cakir M, Grossman AB. Medullary thyroid cancer: molecular biology and novel molecular therapies. Neuroendocrinology. 2009;90(4):323-348.

5. Hoff AO, Hoff PM. Medullary thyroid carcinoma. Hematol Oncol Clin North Am. 2007;21(3):475-488.

6. Fujita T, Fujimori M. The treatment of medullary thyroid cancer. Gan To Kagaku Ryoho. 2009;36(10):1627-1631. Japanese.

7. Dralle H, Lorenz K, Machens A. Surgery of the thyroid carcinoma. Chirurg. 2009;80(11):1069-1082. German.

8. Bagaturia GO. Combined surgical interventions for advanced thyroid carcinoma. Vestn Khir Im I I Grek. 2009;168(5):31-34. Russian.

9. Rodriguez-Antona C, Pallares J, Montero-Conde C, et al. Overexpression and activation of EGFR and VEGFR2 in medullary thyroid carcinomas is related to metastasis. Endocr Relat Cancer. 2010;17(1): $7-16$.

10. Woyach JA, Shah MH. New therapeutic advances in the management of progressive thyroid cancer. Endocr Relat Cancer. 2009;16(3): 715-731.

11. National Comprehensive Cancer Network. NCCN Clinical Practice Guidelines in Oncology. Jenkintown, PA: National Comprehensive Cancer Network; 2009.

12. Higgins MJ, Forastiere A, Marur S. New directions in the systemic treatment of metastatic thyroid cancer. Oncology (Williston Park). 2009;23(9):768-775.

13. Cerrato A, De Falco V, Santoro M. Molecular genetics of medullary thyroid carcinoma: the quest for novel therapeutic targets. $J \mathrm{Mol}$ Endocrinol. 2009;43(4):143-155.

14. Fassnacht M, Kreiss1 MC, Weismann D, Allolio B. New targets and therapeutic approaches for endocrine malignancies. Pharmacol Ther. 2009;123(1):117-141.

15. Sherman SI. Advances in chemotherapy of differentiated epithelial and medullary thyroid cancers. J Clin Endocrinol Metab. 2009;94(5): 1493-1499. 
16. Lanzi C, Cassinelli G, Nicolini V, et al. Targeting RET for thyroid cancer therapy. Biochem Pharmacol. 2009;77(3):297-309.

17. Ye L, Santarpia L, Gagel RF. Targeted therapy for endocrine cancer: the medullary thyroid carcinoma paradigm. Endocr Pract. 2009; 15(6):597-604.
18. Orphanos GS, Ioannidis GN, Ardavanis AG. Cardiotoxicity induced by tyrosine kinase inhibitors. Acta Oncol. 2009;48(7):964-970.

\section{Publish your work in this journal}

The International Medical Case Reports Journal is an international, peer-reviewed open-access journal publishing original case reports from all medical specialties. Previously unpublished medical posters are also accepted relating to any area of clinical or preclinical science. Submissions should not normally exceed 2,000 words or
4 published pages including figures, diagrams and references. The manuscript management system is completely online and includes a very quick and fair peer-review system, which is all easy to use. Visit $\mathrm{http}: / / \mathrm{www}$.dovepress.com/testimonials.php to read real quotes from published authors.

Submit your manuscript here: http://www.dovepress.com/international-medical-case-reports-journal-journal 